\title{
Endoscopic epiglottopexy using Lichtenberger's needle carrier to avoid breakdown of repair
}

\author{
Kishore Sandu $^{1} \cdot$ Philippe Monnier $^{1} \cdot$ Antoine Reinhard $^{1} \cdot$ Francois Gorostidi $^{1}$
}

Received: 3 April 2015/Accepted: 30 June 2015/Published online: 11 July 2015

(C) Springer-Verlag Berlin Heidelberg 2015

\begin{abstract}
Severe type III laryngomalacia LM is represented by a retroflexed epiglottis that touches the posterior pharyngeal wall and obstructs the laryngeal inlet. Endoscopic epiglottopexy is advised in such cases wherein pexy sutures are passed between the epiglottis and base of tongue. Using conventional needle carriers, it is difficult to pass such sutures that go deep enough into the tongue base. Such a pexy is prone to a break down. We describe a novel technique of placing these glossoepiglottic sutures using the Lichtenberger's needle carrier. We used this technique in three patients with excellent results and report no complications. We propose to use this technique in cases of epiglottic prolapse seen in severe LM and certain hypotonic conditions.
\end{abstract}

Keywords Endoscopic epiglottopexy $\cdot$ Severe laryngomalacia $\cdot$ Lichtenberger's needle carrier

\section{Introduction}

Laryngomalacia (LM) is the most common cause of stridor in small children accounting for around $60 \%$ of all congenital anomalies of the larynx. Morphologically there are three types: floppy supra arytenoid tissue in type I, short aryepiglottic folds and omega-shaped epiglottis in type II,

Electronic supplementary material The online version of this article (doi:10.1007/s00405-015-3707-5) contains supplementary material, which is available to authorized users.

Kishore Sandu

kishore.sandu@chuv.ch

1 Department of Otolaryngology, University Hospital, Lausanne, Switzerland and retroflexed epiglottis in type III. The exact cause for LM is unknown, though several theories have been proposed. Immaturity of laryngeal cartilages could be a contributing factor, though it has never been proved histologically [1]. Thompson et al. [2] showed that the floppiness of laryngomalacia tissues could be related to an altered laryngeal tone and sensorimotor integrative functions. This can explain both-the stridor and occasional feeding difficulties that are seen associated with LM, but not the three morphological types usually described. Anatomical variants of the epiglottis also play a distinct role in this pathology.

Retroflexed epiglottis that touches the posterior pharyngeal wall represents the severest form of LM [3]. Such a prolapsed epiglottis can present with symptoms of obstructive sleep apnea and can be associated with upper airway hypotonia. Surgical treatment of such an abnormal epiglottis has been described and essentially consists of creating a raw area (either with laser or electrocautery) in the vallecula and adjoining tongue base and then placing pexy sutures attaching the epiglottis to the tongue [4, 5]. Oomen et al. [5] used the $\mathrm{CO}_{2}$ laser and a curved alligator forceps to transorally fix the epiglottis with the base of tongue in five patients, though the authors do not mention their intra-, post-operative and long-term experience. Our own experience in such cases has presented with following problems:

1. Sutures passing superficially into the tongue mucosa (even if multiple such sutures have been passed) are prone to breakdown and cause recurrence of symptoms. For the glossoepiglottic sutures to be successful, they need to pass sufficiently deep into the tongue substance.

2. Placing sutures endoscopically between the epiglottis and substance of the tongue base is not very easy. 
Manipulating conventional suture needles to get sufficiently into the base of tongue is difficult.

\section{Materials and methods}

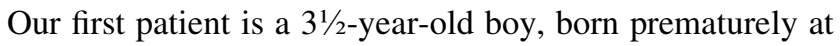
27 weeks and weighed $800 \mathrm{~g}$ at birth. He had severe bronchopulmonary dysplasia and underwent bilobectomy for lung empyma at 3 months of age at a different institution outside Switzerland. During this prolonged hospital stay, subsequently, he underwent a tracheostomy at 4 months of age for prolonged intubation-induced grade III subglottic stenosis SGS. He was then referred to our institution for further management.

The child was slightly undernourished and had delayed neurological development. A feeding gastrostomy was categorically refused by the parents, and hence he was fed by a nasogastric tube. Awake and sleep endoscopy showed large adenoids and retroflexed epiglottis touching the posterior pharyngeal wall that completely occluded the laryngeal inlet (type III LM). There was no glossoptose. He had normal mobility of the vocal cords, severe grade III SGS and mild to moderate degree of tracheobronchomalacia. Following stepwise management was planned for our patient:

Step 1 Adequate medical management for the reactive pharyngolarynx.

Step 2 Adenoidectomy and endoscopic epiglottopexy.

Step 3 Cricotracheal resection for the SGS.

In the following months, the child had two failed attempts of epiglottopexy using conventional needle carriers. We feel, these were due to the fact that our sutures were not passed deep enough into the tongue substance. The sutures could not hold on to the epiglottis which continued to be in the retroflexed position and causing significant laryngeal obstruction.

The second patient is an 11-year-old girl, born prematurely at 27 weeks of gestation having retarded psychomotor development. She had undergone a cricotracheal resection for an intubation-induced grade IV SGS at 1 year of life. She had a floppy retroflexed epiglottis that became significantly hypotonic in compromising her airway some years after the surgery.

Our third patient is a 3-month-old girl who had two prior failed supraglottoplasties for severe LM at another institution. She continued having stridor and feeding difficulties and was then referred to us for treatment. The previous attempts had shortened the aryepiglottic folds due to cicatricial contraction that pulled back the epiglottis- further retroflexing it and leading to an early supraglottic stenosis.

\section{Modified technique of epiglottopexy EP}

Informed and written consent of the parents of all three patients was obtained. The technique was cleared by the scientific committee of our hospital.

Anesthesia is given through the tracheostomy canula (first case) and through a nasotracheal tube in the other two cases. An adequate size Benjamin-Lindholm BL or Parsons (with a side slot) laryngoscope is used to suspend the larynx. Adequate passing of the Lichtenberger needle carrier through the BL laryngoscope is checked before the suspension laryngoscopy. The spatula of the laryngoscope is inserted to expose the tongue base, entire valleculae and epiglottis. Using the Lumenis $\mathrm{CO}_{2}$ laser (continuous mode, $3 \mathrm{~W}$, defocused beam), the valleculae and adjacent tongue base are demucosalized (Fig. 1). A 2-mm mucosal rim is left at the edge of the epiglottis. A wet cotton swab is used to remove the charred tissue and confirm that no normal mucosal island is left behind. This is done to ensure an adequate cicatricial attachment and avoid a retention cyst

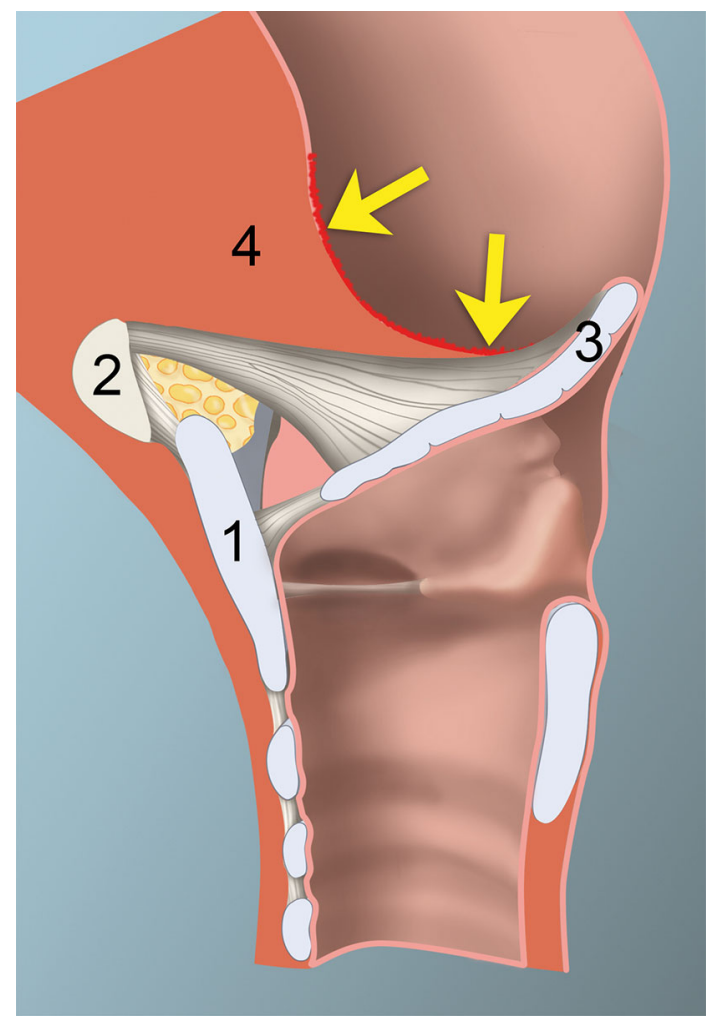

Fig. 1 Laser vaporization of the valleculae and adjacent base of tongue. 1 Thyroid cartilage, 2 hyoid bone, 3 epiglottis, 4 base of tongue 
formation in the future when the two surfaces stick to each other.

Using 3-0 vicryl on Lichtenberger's needle carrier (Richard Wolf D-78532 Knittlingen, Germany), two lateral and vertical transfixiant sutures are passed transorally from back to front toward the skin, to attach the raw surfaces of the epiglottis and tongue base. The first stitch is passed on the right side of the epiglottis so as to fix this portion of the epiglottis, then into the tongue base substance, emerging outside in the neck (Fig. 2). The other limb of this suture is then passed at somewhat higher level on the right side of the epiglottis. The contralateral suture is passed in a similar fashion through the left portion of the epiglottis. These sutures are vertical and slightly oblique (Fig. 3). Both the sutures are placed above the hyoid bone, about $3 \mathrm{~mm}$ within the free edge of the epiglottis and are far from the hypoglossal nerves. The two vicryl sutures are buried in the subcutaneous tissue by a small neck incision (Fig. 4). The entire procedure takes about $20 \mathrm{~min}$, and the patient is given adequate post-operative analgesics and broad-spectrum antibiotics. Patients 2 and 3 were extubated at the end of the procedure and required noninvasive ventilation by mask for 24-72 h. After checking for normal tongue movements, deglutition exercises with a pediatric swallow therapist are started within a couple of days. On checking endoscopy at 1 month shows the epiglottis sticking well to the tongue base and the laryngeal inlet optimally open, Fig. 5.

The third case with an early supraglottic stenosis is interesting. Using the $\mathrm{CO}_{2}$ laser in the ultrapulse mode, the aryepiglottic folds were cautiously divided on both sides. The epiglottis was mobilized anteriorly, and fixed with the

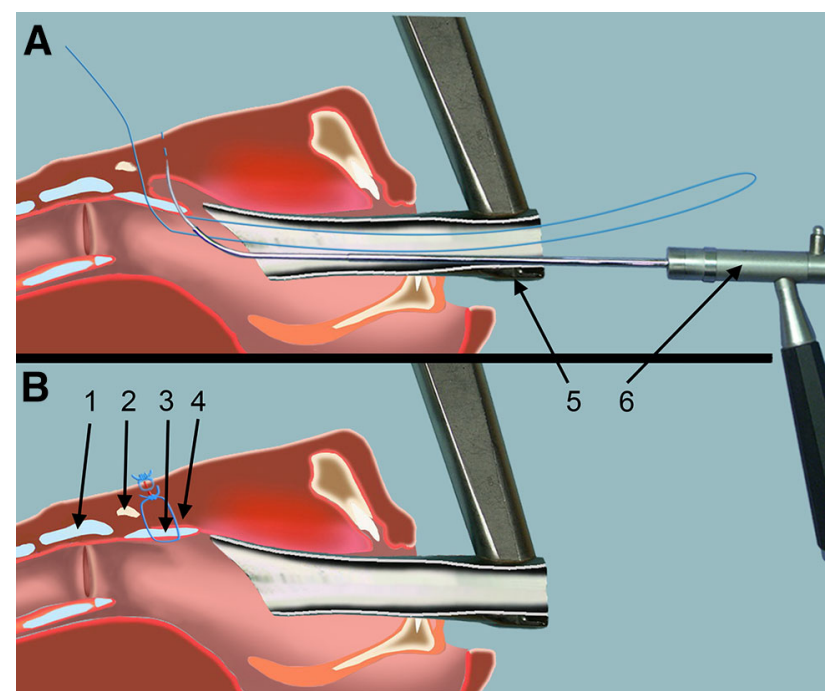

Fig. 2 a Passing of two transfixiating sutures using the Lichtenberger needle carrier. b Tying of the sutures in the subcutaneous tissue. 1 Thyroid cartilage, 2 hyoid bone, 3 epiglottis, 4 base of tongue, 5 Benjamin-Lindholm laryngoscope, 6 Lichtenberger needle carrier

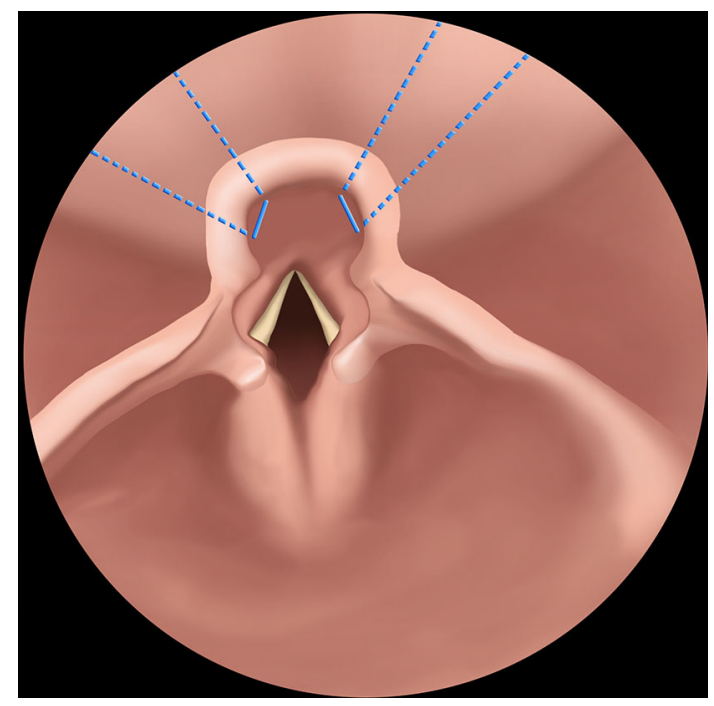

Fig. 3 Two transfixiant vertical and slightly oblique glossoepiglottic sutures

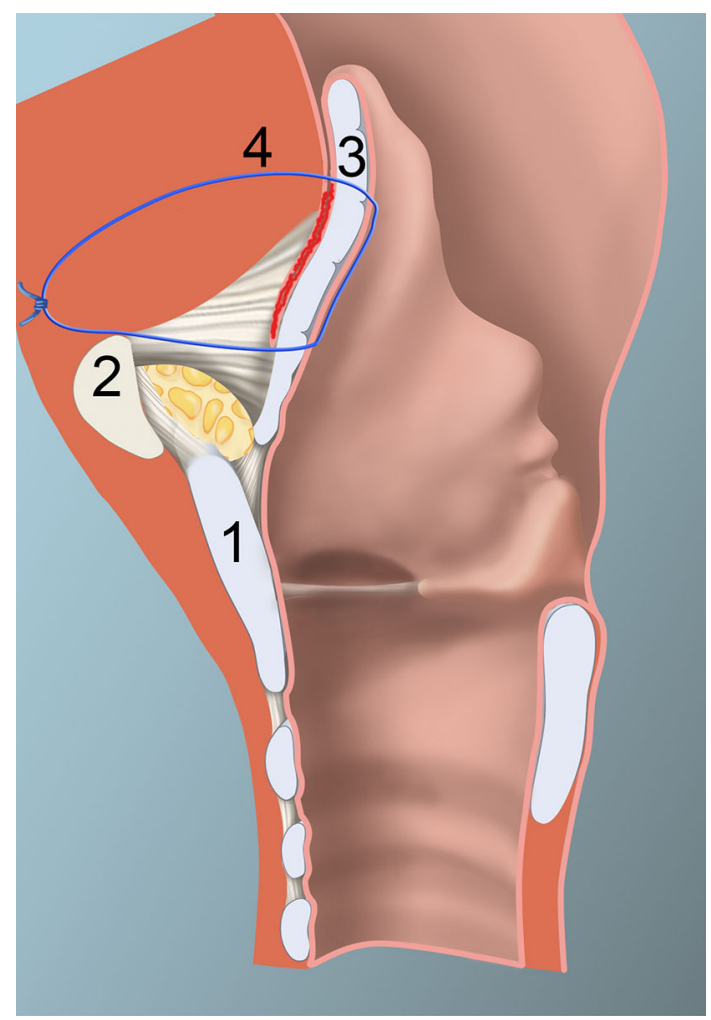

Fig. 4 Glossoepiglottic pexy sutures holding the epiglottis upright against the base of tongue. 1 Thyroid cartilage, 2 hyoid bone, 3 epiglottis, 4 base of tongue

base of tongue using the technique described earlier. The paraglottic and piriform sinus mucosae were mobilized and sutured anterolaterally up to the vestibular folds. The endoscopic sutures were placed using 5.0 vicryl to cover the raw surface created during division of the aryepiglottic 
Fig. 5 a Pre-operative transnasal flexible endoscopic view of the retroflexed prolapsed epiglottis touching the posterior pharyngeal wall. b Post-operative transnasal flexible endoscopic view of the epiglottis sutured to base of tongue and the laryngeal inlet completely open

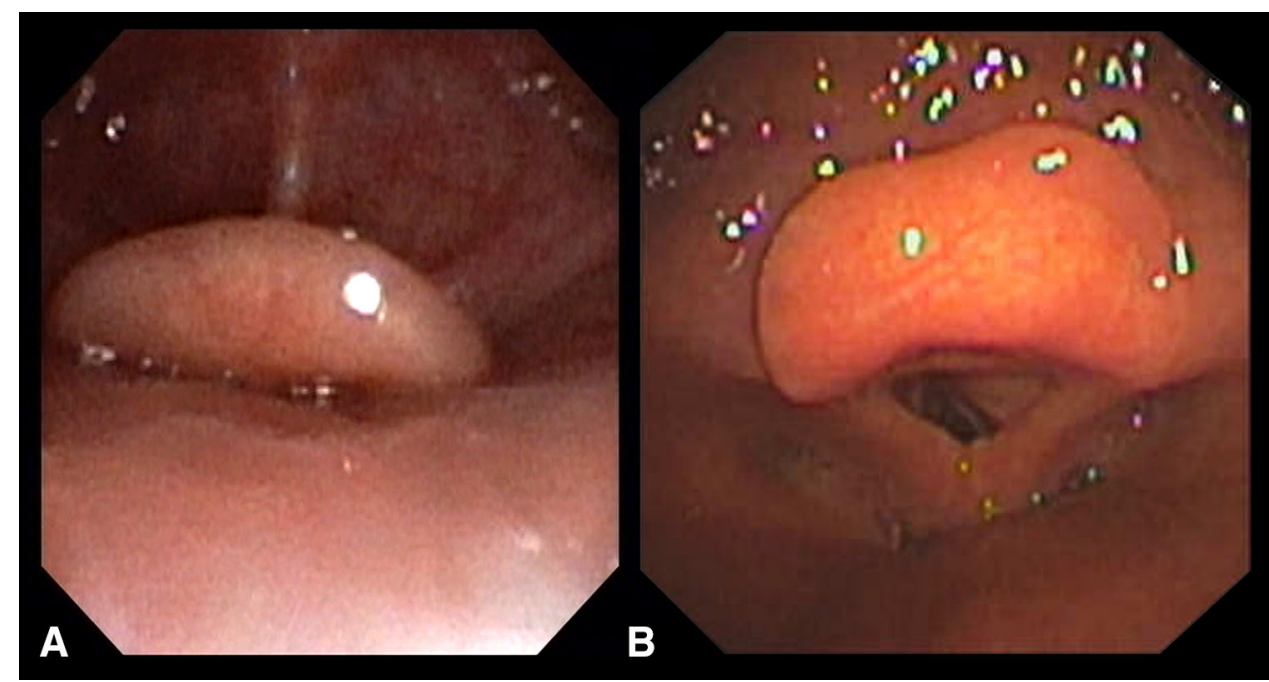

folds with mucosa. The fully mucosalized supraglottis prevented the pulling back of the epiglottis during the cicatrization process.

The first child subsequently had a cricotracheal resection and presently is decannulated. At 6-month follow-up, all three patients are symptom-free, have no feeding difficulties and record an age-appropriate growth. The most recent flexible endoscopy of all the three patients during spontaneous respiration shows that the epiglottis in an upright position and the laryngeal inlet adequately open. There was no hypoglossal nerve trauma in any of the patients.

\section{Discussion}

A retroflexed epiglottis as in the severe form (type III) of LM touches the posterior pharyngeal wall and obstructs the laryngeal inlet. This may cause respiratory symptoms-stridor, apneic spells and feeding difficulties. Supraglottoplasty SGP is the most commonly performed surgical intervention for symptomatic LM types I and II. However, this surgery is insufficient in type III LM [4, 5]. The objective in treating such a severe form of LM is to attach the epiglottis with the base of tongue so as to adequately open the laryngeal inlet. The valleculae and adjacent tongue base are scarified either using the laser or electrocautery and pexy sutures thrown to stick the two surfaces together. Placing such sutures can be cumbersome, time consuming and requires expertise. Only when these sutures are taken through the epiglottic cartilage and passed deep into the tongue substance, they can be successful in attaching the two raw surfaces to each other. The tongue is a muscular structure and moves constantly during swallowing and speech. Sutures that are placed superficially into the tongue will open up in a few days and not serve the purpose. An epiglottic suture has been described by Fajdiga et al. [6] to correct the tubular shape of the epiglottis (LM type II). They remove a strip of mucosa on the lingual surface of the epiglottis and place a resorbable stitch transversely to uncurl the bent cartilage. Though, their technique is not suited for a retroflexed epiglottis. Whymark et al. [7] used the $\mathrm{CO}_{2}$ laser to induce scar tissue in the valleculae to roll up the epiglottis, without actually placing the pexy sutures. They used this technique in 76 patients based on symptoms and not on the morphology type of LM. They report revision surgery in $12 \%(7 / 76)$ cases. Clinical and symptomatic improvement before and after SGP and epiglottopexy can be objectivated using polysomnography [8, 9] and growth percentile graphs [10].

In patients with multiple levels of airway obstructions, it is imperative to proceed in a stepwise manner to avoid decannulation failures. The surgical management of our patients was done only after they had received adequate medical management for their reactive larynges.

In our technique of epiglottopexy EP, two vertical and slightly oblique glossoepiglottic sutures are placed using the Lichtenberger's needle carrier. The sutures are passed transorally from back to front through the epiglottis, deep into the tongue base and emerging out in the neck. The sutures are tied and buried subcutaneously, after verifying the exact position of the epiglottis in relation to the larynx. In the endoscopic approach, our operating position is axial to the oropharynx and the laryngeal inlet. Thus, these sutures can be correctly placed to improve the airway morphology and the patient's symptoms. In addition, it is 
quick, simple and does not require special expertise. We never had a problem in passing the Lichtenbergers' needle carrier through a closed Benjamin-Lindholm laryngoscope. But in the event of a no or difficult passage, we suggest to switch to a Parsons laryngoscope with a side slot that will allow better maneuvering of the needle carrier.

Conventional needle carriers may not always allow the suture needles to pass deep into the tongue base. This will cause surgical failures as had happened in previous attempts in two of our patients. The Lichtenberger's needle carrier allows easy maneuvering and passing the sutures well into the tongue base substance. Also, these sutures are well within the tongue midline and far from the lingual vessels and hypoglossal nerves. The patients require swallow therapy and resume normal feeds within couple of days. None of our patients had dysphagia or bronchoaspiration after placing the pexy sutures.

Supraglottic stenosis is the most dreaded complication after SGP and around $4 \%$ incidence has been reported [11, 12]. Reddy et al. [12] proposed a unilateral SGP to reduce the incidence of supraglottic stenosis, though a contralateral procedure had to be redone in $17 \%$ of their cases. Preciado et al. [13] found SGP failures associated with comorbidities (cardiac, neurological) and severe gastroesophageal reflux. It is unclear from the published medical evidence whether LM severity alone would portend a higher risk of SGP surgical failure. Multi-operated supraglottoplasties may lead to development of a supraglottic stenosis and should be managed with utmost caution. Our third patient is an example of such a situation. Incorrect and excessive mucosal resection of the aryepiglottic folds will lead to cicatricial contraction and pulling back of the epiglottis leading to supraglottic stenosis. In revision SGP, one must be careful and try to cover the supraglottic raw areas using the paraglottic and piriform sinus mucosae. This will reduce the fibrosis-induced pulling back and further retroflexion of the epiglottis. Attempts must be made to completely mucosalize the supraglottis at the end of the surgery with very little or no raw area left behind that would granulate and cause cicatricial stenosis.

We strongly feel that the EP with pexy sutures should be reserved for an obvious epiglottic retroflexion (type III LM). The technique of SGP should be selected as per the morphology type of LM based on a dedicated pre-operative assessment. The use of tools (whether using cold instruments/laser/microdebrider) to correct the supraglottis is based on the operator's choice, training and his or her surgical comfort. All present techniques of SGP and EP can achieve good results-their main aims should be symptom relief and avoidance of complications.

We propose to use our modified technique of EP in the following indications:
1. Type III LM.

2. Obstructive sleep apnea and certain hypotonic conditions with epiglottic overhang causing laryngeal obstruction.

3. Supraglottic stenosis (EP with re-mucosalisation of the supraglottic raw areas).

Long-term results, larger number of patients and prospective studies using pre- and post-operative polysomnography and growth percentiles in these cases of severely retroflexed epiglottis would be interesting to see in future studies.

\section{Conclusion}

Severe type III LM with retroflexed epiglottis will require pexy sutures between the epiglottis and the base of tongue. Placing these sutures can be cumbersome and time consuming. In addition, sutures passing superficially through the tongue are prone to breakdown. The Lichtenberger's needle carrier allows correct placement of transfixion stapler-like pexy sutures and guarantees optimal results.

Conflict of interest The authors declare that they have no conflict of interest.

\section{References}

1. Lane RW, Weider DJ, Steinem C (1984) Laryngomalacia: a review and case report of surgical treatment with resolution of pectus excavatum. Arch Otolarynol 110:546-551

2. Thompson DM (2007) Abnormal sensorimotor integrative function of the larynx in congenital laryngomalacia: a new theory of etiology. Laryngoscope 117:1-33

3. Holinger LD, Lusk RP, Green CG (1997) Pediatric laryngology and bronchoesophagology, 139-142. Lippincott-Raven, Philadelphia, New York

4. Werner JA, Lippert BM, Dünne AA, Ankermann T, Folz BJ, Seyberth H (2002) Epiglottopexy for the treatment of severe laryngomalacia. Eur Arch Otorhinolaryngol 259(9):459-464

5. Oomen K, Modi VK (2014) Epiglottopexy with and without lingual tonsillectomy. Laryngoscope 124:1019-1022

6. Fajdiga I, Beden AB, Krivec U, Iglic Č (2008) Epiglottic suture for treatment of laryngomalacia. Intl J Ped Otorhinolaryngol $72: 1345-1351$

7. Whymark AD, Clement WA, Kubba H, Geddes NK (2006) Laser epiglottopexy for laryngomalacia: 10 years' experience in the west of Scotland. Arch Otolaryngol Head Neck Surg 132:978-982

8. Sesterhenn AM, Zimmermann AP, Bernhard M, Kussin A, Timmesfeld N, Stiller S, Wiegand S, Maier RF, Werner JA (2009) Polysomnography outcomes following transoral $\mathrm{CO}_{2}$ laser microsurgery in pediatric patients with laryngomalacia. Int J Ped Otorhinolaryngol 73:1339-1343 
9. O'Connor TE, Bumbak P, Vijayasekaran S (2009) Objective assessment of supraglottoplasty outcomes using polysomnography. Int J Pediatr Otorhinolaryngol 73(9):1211-1216

10. Meier JD, Nguyen SA, White DR (2011) Improved growth curve measurements after supraglottoplasty. Laryngoscope 121(7):1574-1577

11. Denoyelle F, Mondain M, Gresillon N, Roger G, Chaudre F, Garabedian EN (2003) Failures and complications of supraglottoplasty in children. Arch Otolaryngol Head Neck Surg 129(10):1077-1080

12. Reddy DK, Matt BH (2001) Unilateral versus bilateral supraglottoplasty for severe laryngomalacia in children. Arch Otol Head Neck Surg 127:694-699

13. Preciado D, Zalzal G (2012) A systematic review of supraglottoplasty outcomes. Arch Otolaryngol Head Neck Surg 138(8):718-721 